Research Article

\title{
Global Convergence of a Modified Two-Parameter Scaled BFGS Method with Yuan-Wei-Lu Line Search for Unconstrained Optimization
}

\author{
Pengyuan Li, ${ }^{1}$ Zhan Wang, ${ }^{1}$ Dan Luo $\left(\mathbb{D},{ }^{2}\right.$ and Hongtruong Pham ${ }^{3}$ \\ ${ }^{1}$ College of Mathematics and Information Science, Guangxi University, Nanning, Guangxi, China \\ ${ }^{2}$ School of Mathematics and Statistics, Baise University, Baise, Guangxi, China \\ ${ }^{3}$ Thai Nguyen University of Economics and Business Administration, Thai Nguyen, Vietnam
}

Correspondence should be addressed to Dan Luo; bsxyldan@163.com

Received 23 July 2020; Accepted 5 August 2020; Published 26 August 2020

Guest Editor: Weijun Zhou

Copyright (c) 2020 Pengyuan Li et al. This is an open access article distributed under the Creative Commons Attribution License, which permits unrestricted use, distribution, and reproduction in any medium, provided the original work is properly cited.

The BFGS method is one of the most efficient quasi-Newton methods for solving small- and medium-size unconstrained optimization problems. For the sake of exploring its more interesting properties, a modified two-parameter scaled BFGS method is stated in this paper. The intention of the modified scaled BFGS method is to improve the eigenvalues structure of the BFGS update. In this method, the first two terms and the last term of the standard BFGS update formula are scaled with two different positive parameters, and the new value of $y_{k}$ is given. Meanwhile, Yuan-Wei-Lu line search is also proposed. Under the mentioned line search, the modified two-parameter scaled BFGS method is globally convergent for nonconvex functions. The extensive numerical experiments show that this form of the scaled BFGS method outperforms the standard BFGS method or some similar scaled methods.

\section{Introduction}

Consider

$$
\min f(x) \text {, }
$$

where $x \in \mathfrak{R}^{n}$, and $f: \mathfrak{R}^{n} \longrightarrow \mathfrak{R}$ is a continuously differentiable function bounded from below. The quasi-Newton methods are currently used in countless optimization software for solving unconstrained optimization problems [1-8]. The BFGS method, one of the most efficient quasi-Newton methods, for solving (1) is an iterative method of the following form:

$$
x_{k+1}=x_{k}+\alpha_{k} d_{k} \text {, }
$$

where $k=0,1,2, \ldots, \alpha_{k}$, obtained by some line search rule, is a step size, and $d_{k}$ is the BFGS search direction computed by the following equation:

$$
B_{k} d_{k}=-g_{k},
$$

where $g_{k}=g\left(x_{k}\right)$ is the gradient of $f(x)$, and the matrix $B_{k}$ is the BFGS approximation to the Hessian $\nabla^{2} f\left(x_{k}\right)$, which has the following update formula:

$$
B_{k+1}=B_{k}-\frac{B_{k} s_{k} s_{k}^{T} B_{k}}{s_{k}^{T} B_{k} s_{k}}+\frac{y_{k} y_{k}^{T}}{y_{k}^{T} s_{k}},
$$

where $s_{k}=x_{k+1}-x_{k}$ and $y_{k}=g_{k+1}-g_{k}$. The problems related to the BFGS method have been analyzed and studied by many scholars, and satisfactory conclusions have been drawn [9-16]. In earlier year, Powell [17] first proved the global convergence of the standard BFGS method with inexact Wolfe line search for convex functions. Under the exact line search or some specific inexact line search, the BFGS method has the convergence property for convex minimization problems [18-21]. By contrast, for nonconvex problems, Mascaren [22] has presented an example to elaborate that the BFGS method and some Broyden-type methods may not be convergent under the exact line search. As such, with the Wolfe line searches, Dai [23] also proved that the BFGS method may fail to converge. To verify the global convergence of the BFGS method for general functions and to obtain a better Hessian approximation matrix of the objective function, Yuan and Wei [24] presented a modified quasi-Newton equation as follows: 


$$
B_{k+1} s_{k}=\hat{y}_{k}
$$

where

$$
\begin{aligned}
& \widehat{y}_{k}=y_{k}+\frac{\max \left\{C_{k}, 0\right\}}{\left\|s_{k}\right\|^{2}} s_{k}, \\
& C_{k}=2\left[f\left(x_{k}\right)-f\left(x_{k}+\alpha_{k} d_{k}\right)\right]+\left(g\left(x_{k}+\alpha_{k} d_{k}\right)+g\left(x_{k}\right)\right)^{T} s_{k},
\end{aligned}
$$

$B_{k+1}=B_{k}-\frac{B_{k} s_{k} s_{k}^{T} B_{k}}{s_{k}^{T} B_{k} s_{k}}+\frac{\widehat{y}_{k} \widehat{y}_{k}^{T}}{s_{k}^{T} \widehat{y}_{k}}$.

In practice, the standard BFGS method has many qualities worth exploring and can effectively solve a class of unconstrained optimization problems.

Here, two excellent properties of the BFGS method are introduced. One is the self-correcting quality, scilicet; if the current Hessian approximate inverse matrix estimates the curvature of the function incorrectly, then Hessian approximation matrix $H_{k}$ will correct itself within a few steps. The other interesting property is that small eigenvalues are better corrected than large ones [25]. Hence, one can see that, the efficiency of the BFGS algorithm is subject to the eigenvalues structure of the Hessian approximation matrix intensely. To improve the performances of the BFGS method, Oren and Luenberger [26] scaled the Hessian approximation matrix $B_{k}$, that is, they replaced $B_{k}$ by $\tau_{k} B_{k}$, where $\tau_{k}$ is a self-scaling factor. Nocedal and Yuan [27] further studied the self-scaling BFGS method when $\tau_{k}=\left(y_{k}^{T} s_{k} / s_{k}^{T} B_{k} s_{k}\right)$. Based on the value of this $\tau_{k}$, Al-Baali [28] introduced a simple modification: $\tau_{k}=\min \left\{1, \tau_{k}\right\}$. The numerical experiments showed that the modified self-scaling BFGS method outperforms the unscaled BFGS method. Many other scaled BFGS methods with better properties will be enumerated.

Formula 1. The general one-parameter scaled BFGS updating formula is

$$
B_{k+1}=B_{k}-\frac{B_{k} s_{k} s_{k}^{T} B_{k}}{s_{k}^{T} B_{k} s_{k}}+\gamma_{k} \frac{y_{k} y_{k}^{T}}{y_{k}^{T} s_{k}},
$$

where $\gamma_{k}$ is a positive parameter, and it is diverse for the selection of the scaled factor $\gamma_{k}$, which is listed as follows.

Choice A:

$$
\gamma_{k}=\frac{2}{y_{k}^{T} s_{k}}\left(f\left(x_{k}\right)-f\left(x_{k+1}\right)+s^{T} g_{k+1}\right),
$$

where the value of $\gamma_{k}$ is given by Yuan [29], and with inexact line search, the global convergence of the scaled BFGS method with $\gamma_{k}$ given by (9) is established for convex functions by Powell [30]. Ulteriorly, for general nonlinear functions, Yuan limited the value range of $\gamma_{k}$ to $[0.01,100]$ to ensure the positivity of $\gamma_{k}$ under the inexact line search and proved the global convergence of the scaled BFGS method in this form.

Choice B:

$$
\gamma_{k}=\frac{y_{k}^{T} s_{k}}{\left\|y_{k}\right\|^{2}}
$$

which is obtained as a solution of the problem: $\min \left\|s_{k}-\gamma_{k} y_{k}\right\|^{2}$. The scaled BFGS method based on this value of $\gamma_{k}$ was introduced by Barzilai and Borwein [31] and was deemed the spectral scaled BFGS method. Cheng and $\mathrm{Li}$ [32] proved that the spectral scaled BFGS method is globally convergent under Wolfe line search with assuming the convexity of the minimizing function.

Choice C:

$$
\gamma_{k}=\min \left\{\frac{y_{k}^{T} s_{k}}{\left\|y_{k}\right\|^{2}+\beta_{k}}, 1\right\},
$$

where $\beta_{k}>0$ for $k=0,1, \ldots$. Under the Wolfe line search (20) and (21), $y_{k}^{T} s_{k}>0$ holds for $k=0,1, \ldots$, which implies that $\gamma_{k}$ computed by (11) is bounded away from zero, that is to say, $0<\gamma_{k} \leq 1$. Therefore, in this instance, the large eigenvalues of $B_{k+1}$ given by (8) are shifted to the left [33].

Formula 2. Proposed by Oren and Luenberger [26], this scaled BFGS method was the single parameter scaled of the first two items of the BFGS update and was defined as

$$
B_{k+1}=\delta_{k}\left[B_{k}-\frac{B_{k} s_{k} s_{k}^{T} B_{k}}{s_{k}^{T} B_{k} s_{k}}\right]+\frac{y_{k} y_{k}^{T}}{y_{k}^{T} s_{k}},
$$

where $\delta_{k}$ is a positive parameter and is calculated as follows:

$$
\delta_{k}=\frac{y_{k}^{T} s_{k}}{s_{k}^{T} B_{k} s_{k}}
$$

The parameter $\delta_{k}$ assigned by (13) can make the structure of eigenvalue to inverse Hessian approximation more easily analyzed. Consequently, it is regarded as one of the best factors.

Formula 3. In this method, the scaled parameters are selected to cluster the eigenvalues of the iteration matrix $B_{k+1}$ and shift the large eigenvalues to the left. The update formula of the Hessian approximate matrix is computed as

$$
B_{k+1}=\delta_{k}\left[B_{k}-\frac{B_{k} s_{k} s_{k}^{T} B_{k}}{s_{k}^{T} B_{k} s_{k}}\right]+\gamma_{k} \frac{y_{k} y_{k}^{T}}{y_{k}^{T} s_{k}},
$$

where both $\delta_{k}$ and $\gamma_{k}$ are positive parameters, and Andrei [34] preset them as the following values:

$$
\begin{aligned}
& \gamma_{k}=\min \left\{\frac{y_{k}^{T} s_{k}}{\left\|y_{k}\right\|^{2}+\left|s^{T} g_{k+1}\right|}, 1\right\}, \\
& \delta_{k}=\frac{n-\gamma_{k}\left(\left\|y_{k}\right\|^{2} / y_{k}^{T} s_{k}\right)}{n-\left(\left\|B_{k} s_{k}\right\|^{2} / s_{k}^{T} B_{k} s_{k}\right)} .
\end{aligned}
$$

If the scaled parameters are bounded and line search is inexact, then this scaled BFGS algorithm is globally convergent for general functions. A large number of numerical experiments show that the double parameter scaled BFGS 
method with $\delta_{k}$ and $\gamma_{k}$ given by (15) and (16) is more competitive than the standard BFGS method. In this paper, combining (7) and (14), we propose a new update formula of $B_{k+1}$ listed as follows:

$$
B_{k+1}=\delta_{k}\left[B_{k}-\frac{B_{k} s_{k} s_{k}^{T} B_{k}}{s_{k}^{T} B_{k} s_{k}}\right]+\gamma_{k} \frac{\widehat{y}_{k} \widehat{y}_{k}^{T}}{\hat{y}_{k}^{T} s_{k}},
$$

where $\widehat{y}_{k}$ is determined by formula (6),

$$
\begin{aligned}
\gamma_{k} & =\frac{\widehat{y}_{k}^{T} s_{k}}{\left\|\widehat{y}_{k}\right\|^{2}+\left\|s_{k}\right\|^{2}}, \\
\delta_{k} & =\frac{n-\gamma_{k}\left(\left\|\widehat{y}_{k}\right\|^{2} / \widehat{y}_{k}^{T} s_{k}\right)}{n-\left(\left\|B_{k} s_{k}\right\|^{2} / s_{k}^{T} B_{k} s_{k}\right)} .
\end{aligned}
$$

Some interesting properties of the BFGS-type method are inseparable from the weak Wolfe-Powell (WWP) line search:

$$
\begin{aligned}
& f\left(x_{k}+\alpha_{k} d_{k}\right) \leq f_{k}+\xi \alpha_{k} g_{k}^{T} d_{k}, \\
& g\left(x_{k}+\alpha_{k} d_{k}\right)^{T} d_{k} \geq \sigma g_{k}^{T} d_{k},
\end{aligned}
$$

where $0<\xi<\sigma<1$. There are many research studies based on this line search [35-43]. To further develop the inexact line search, Yuan et al. present a new line search and call it Yuan-Wei-Lu (YWL) line search, which has the following form:

$$
\begin{gathered}
f\left(x_{k}+\alpha_{k} d_{k}\right) \leq f_{k}+\xi \alpha_{k} g_{k}^{T} d_{k} \\
+\alpha_{k} \min \left[-\xi_{1} g_{k}^{T} d_{k}, \xi \frac{\alpha_{k}}{2}\left\|d_{k}\right\|^{2}\right], \\
g\left(x_{k}+\alpha_{k} d_{k}\right)^{T} d_{k} \geq \sigma g_{k}^{T} d_{k}+\min \left[-\xi_{1} g_{k}^{T} d_{k}, \xi \alpha_{k}\left\|d_{k}\right\|^{2}\right],
\end{gathered}
$$

where $\xi \in(0,(1 / 2)), \xi_{1} \in(0, \xi)$, and $\sigma \in(\xi, 1)$. The main work of this paper is to verify the global convergence of the modified scaled BFGS update (17) with $\gamma_{k}$ and $\delta_{k}$ given by (18) and (19), respectively, under this line search. Abundant numerical results show that such a combination is appropriate for nonconvex functions.

Our paper is organized as follows. The motivation and algorithm are introduced in the next section. In Section 3, the convergence analysis of the modified two-parameter scaled BFGS method under Yuan-Wei-Lu line search is established. Section 4 is devoted to show the results of numerical experiments. Some conclusions are stated in the last section.

\section{Motivation and Algorithm}

Two crucial tools for analyzing properties of the BFGS method are the trace and the determinant of the $B_{k+1}$ given by (4). Thus, the corresponding relations are enumerated as follows:

$$
\begin{aligned}
& \operatorname{det}\left(B_{k+1}\right)=\operatorname{det}\left(B_{k}\left(I-\frac{s_{k} s_{k}^{T} B_{k}}{s_{k}^{T} B_{k} s_{k}}+\frac{B_{k}^{-1} y_{k} y_{k}^{T}}{y_{k}^{T} s_{k}}\right)\right), \\
& \operatorname{tr}\left(B_{k+1}\right)=\operatorname{tr}\left(B_{k}\right)-\frac{\left\|B_{k} s_{k}\right\|^{2}}{s_{k}^{T} B_{k} s_{k}}+\frac{\left\|y_{k}\right\|^{2}}{s_{k}^{T} y_{k}} .
\end{aligned}
$$

Applying the following existing relation in the study of Sun and Yuan [44],

$$
\operatorname{det}\left(I+v_{1} v_{2}^{T}+v_{3} v_{4}^{T}\right)=\left(1+v_{1}^{T} v_{2}\right)\left(1+v_{3}^{T} v_{4}\right)-\left(v_{1}^{T} v_{4}\right)\left(v_{2}^{T} v_{3}\right)
$$

where $\quad v_{1}=-s_{k}, \quad v_{2}=\left(B_{k} s_{k} / s^{T} B_{k} s_{k}\right), \quad v_{3}=B_{k}^{-1} y_{k}$, and $v_{4}=\left(y_{k} / y_{k}^{T} s_{k}\right)$; we obtain

$$
\operatorname{det}\left(B_{k+1}\right)=\operatorname{det}\left(B_{k}\right) \frac{y_{k}^{T} s_{k}}{s_{k}^{T} B_{k} s_{k}} .
$$

Obviously, the efficiency of the BFGS method depends on the eigenvalues structure of the Hessian approximation matrix, and the BFGS method is actually more affected by large eigenvalues than by small eigenvalues $[25,45,46]$. It can be seen that the second item on the right side of the formula (25) is negative. Therefore, it produces a shift of the eigenvalues of $B_{k+1}$ to the left. Thus, the BFGS method can modify large eigenvalues. Moreover, the third term on the right hand side of (25) being positive produces a shift of the eigenvalues of $B_{k+1}$ to the right. If this term is large, $B_{k+1}$ may have large eigenvalues too. Therefore, the eigenvalues of the $B_{k+1}$ can be corrected by scaling the corresponding items in (25), which is the main motivation for us to use the scaling BFGS method. In this paper, we scale the first two terms and the last term of the standard BFGS update formula with two different positive parameters and propose a new $y_{k}$. In subsequent proof, we will propose some lemmas based on these two important tools to analyze the convergence of the modified scaled BFGS method. Then, an algorithm framework for solving the problem (1) will be built in Algorithm 1, which can be designed as

\section{Convergence Analysis}

In Section 3, the global convergence of Algorithm 1 will be established, and the following assumptions are useful in convergence analysis.

\section{Assumption 1}

(i) The level set $\Upsilon=\left\{x \in \mathfrak{R}^{n} \mid f(x) \leq f\left(x_{0}\right)\right\}$ is bounded

(ii) The function $f(x)$ is twice continuously differentiable and bounded from below

Lemma 1. If $B_{k}$ is the positive definite, $\gamma_{k}>0$, and if $\alpha_{k}$ is computed by (22) and (23), then $B_{k+1}$ given by (17) is an equally positive definite for all $k$. 
Step 1: given an initial point $x_{0} \in \mathfrak{R}^{n}$, an $n \times n$ symmetric positive definite matrix $B_{0}, \varepsilon>0$ is sufficiently small and choose constants $\xi \in(0,(1 / 2)), \xi_{1} \in(0, \xi)$, and $\sigma \in(\xi, 1)$. Set $k=0$.

Step 2: if $\left\|g_{k}\right\| \leq \varepsilon$, stop.

Step 3: obtain a search direction $d_{k}$ by solving $B_{k} d_{k}+g_{k}=0$.

Step 4: compute $\alpha_{k}$ by Yuan-Wei-Lu line search conditions (22) and (23).

Step 5: find the scaling factors $\gamma_{k}$ and $\delta_{k}$ by (18) and (19).

Step 6: let $x_{k+1}=x_{k}+\alpha_{k} d_{k}$. Update $B_{k+1}$ by (17).

Step 7: let $k=k+1$, and go to Step 2 .

Algorithm 1: A modified two parameter scaled BFGS algorithm-MTPSBFGS.

Proof. The inequality (22) and (23) indicates that $y_{k}^{T} s_{k}>0$. Using the definition of $\widehat{y}_{k}$, we obtain

$$
\widehat{y}_{k}^{T} s_{k}=y_{k}^{T} s_{k}+\max \left\{C_{k}, 0\right\} \geq y_{k}^{T} s_{k}>0 .
$$

For any $c \neq 0$,

$$
\begin{aligned}
c^{T} B_{k+1} c & =\delta_{k} c^{T} B_{k} c-\delta_{k} \frac{c^{T} B_{k} s_{k} s_{k}^{T} B_{k} c}{s_{k}^{T} B_{k} s_{k}}+\gamma_{k} \frac{c^{T} \widehat{y}_{k} \widehat{y}_{k}^{T} c}{\widehat{y}_{k}^{T} s_{k}} \\
& =\delta_{k} c^{T} B_{k} c-\delta_{k} \frac{\left(c^{T} B_{k} s_{k}\right)^{2}}{s_{k}^{T} B_{k} s_{k}}+\gamma_{k} \frac{\left(c^{T} \widehat{y}_{k}\right)^{2}}{\widehat{y}_{k}^{T} s_{k}} \\
& \geq \gamma_{k} \frac{\left(c^{T} \widehat{y}_{k}\right)^{2}}{\widehat{y}_{k}^{T} s_{k}}>0,
\end{aligned}
$$

where the penultimate inequality follows, and

$$
\left(s_{k}^{T} B_{k} c\right)^{2} \leq\left(s_{k}^{T} B_{k} s_{k}\right)\left(c^{T} B_{k} c\right), \quad \text { for } z \neq 0,
$$

which is obtained by the Cauchy-Schwarz inequality.

Lemma 2. Let $\delta_{k}$ be generated by (16) for $k=0,1, \ldots$, then $\delta_{k}>0$ and inclines to 1 .

Proof. Observe the formula (19); after substituting $\delta_{k}$, we can find that $\left(\left\|\hat{y}_{k}\right\|^{2} /\left(\left\|\hat{y}_{k}\right\|+\left\|s_{k}\right\|\right)\right)$ is close to 1 . Owing to the symmetry, positive definiteness, and nonsingularity of $B_{k+1}$, its eigenvalues $\lambda_{1}, \ldots, \lambda_{n}$ is real and positive, and $\operatorname{tr}\left(B_{k}\right)=n$. Hence, for $i=1, \ldots, n, \lambda_{i}>0$ and $\sum_{i=1}^{n} \lambda_{i}=n$. Since $\left\|B_{0} s_{0}\right\|^{2}=s_{0}^{T} B_{0} s_{0}, \quad 0<\left\|B_{k} s_{k}\right\|^{2}<1$, and $0<s_{k}^{T} B_{k} s_{k}<1$ for sufficiently large $k,\left\|B_{k} s_{k}\right\|^{2}$, and $s_{k}^{T} B_{k} s_{k}$ are roughly of the same order of magnitude, which shows that $\left(\left\|B_{k} s_{k}\right\|^{2}\right.$ / $\left.s_{k}^{T} B_{k} s_{k}\right) \ll n$. To sum up, the relations $\left(\gamma_{k}\left\|\widehat{y}_{k}\right\|^{2} / \widehat{y}_{k}^{T} s_{k}\right) \ll n$ and $\left(\left\|B_{k} s_{k}\right\|^{2} / s_{k}^{T} B_{k} s_{k}\right) \ll n$ are valid, namely for $k=0,1, \ldots$, $\delta_{k}>0$ and inclines to 1 . The proof is completed.

Remark 1. Based on the conclusion of lemma, we can infer that for any integer $j \in[0, k]$, there exist two positive constants $0<\delta<\Lambda$ satisfying $\delta<\delta_{k} \delta_{k-1}, \ldots, \delta_{j}<\Lambda$.

Lemma 3. If $B_{k+1}$ is updated by (14), where $\gamma_{k}$ and $\delta_{k}$ are determined by (18) and (16), then

$$
\begin{gathered}
\operatorname{tr}\left(B_{k+1}\right) \leq \Lambda \operatorname{tr}\left(B_{0}\right)+\Lambda k+\frac{1}{2}, \\
\sum_{i=0}^{k} \frac{\left\|B_{i} s_{i}\right\|^{2}}{s_{i}^{T} B_{i} s_{i}} \leq \frac{\Lambda}{\delta}\left(\operatorname{tr}\left(B_{0}\right)+k\right)+\frac{1}{2 \delta} .
\end{gathered}
$$

Proof. Considering (25), we have

$$
\begin{aligned}
\operatorname{tr}\left(B_{k+1}\right)= & \delta_{k} \operatorname{tr}\left(B_{k}\right)-\delta_{k} \frac{\left\|B_{k} s_{k}\right\|^{2}}{s_{k}^{T} B_{k} s_{k}}+\gamma_{k} \frac{\left\|\hat{y}_{k}\right\|^{2}}{\widehat{y}_{k}^{T} s_{k}} \\
= & \delta_{k}\left(\delta_{k-1} \operatorname{tr}\left(B_{k-1}\right)-\delta_{k-1} \frac{\left\|B_{k-1} s_{k-1}\right\|^{2}}{s_{k-1}^{T} B_{k-1} s_{k-1}}+\gamma_{k-1} \frac{\left\|\hat{y}_{k-1}\right\|^{2}}{\widehat{y}_{k-1}^{T} s_{k-1}}\right)-\delta_{k} \frac{\left\|B_{k} s_{k}\right\|^{2}}{s_{k}^{T} B_{k} s_{k}}+\gamma_{k} \frac{\left\|\widehat{y}_{k}\right\|^{2}}{\hat{y}_{k}^{T} s_{k}} \\
= & \ldots \\
= & \delta_{k} \delta_{k-1}, \ldots, \delta_{0} \operatorname{tr}\left(B_{0}\right)-\delta_{k} \delta_{k-1}, \ldots, \delta_{0} \frac{\left\|B_{0} s_{0}\right\|^{2}}{s_{T}^{0} B_{0} s_{0}}+\delta_{k} \delta_{k-1}, \ldots, \delta_{1} \gamma_{0} \frac{\left\|\hat{y}_{0}\right\|^{2}}{\hat{y}_{0}^{T} s_{0}} \\
& -\delta_{k} \delta_{k-1}, \ldots, \delta_{1} \frac{\left\|B_{1} s_{1}\right\|^{2}}{s_{1}^{T} B_{1} s_{1}}+\delta_{k} \delta_{k-1}, \ldots, \delta_{2} \gamma_{1} \frac{\left\|\hat{y}_{1}\right\|^{2}}{\hat{y}_{1}^{T} s_{1}} \\
& -\cdots-\delta_{k} \delta_{k-1} \frac{\left\|B_{k-1} s_{k-1}\right\|^{2}}{s_{k-1}^{T} B_{k-1} s_{k-1}}+\delta_{k} \gamma_{k-1} \frac{\left\|\hat{y}_{k-1}\right\|^{2}}{\widehat{y}_{k-1}^{T} s_{k-1}} \\
& -\delta_{k} \frac{\left\|B_{k} s_{k}\right\|^{2}}{s_{k}^{T} B_{k} s_{k}}+\gamma_{k} \frac{\left\|\hat{y}_{k}\right\|^{2}}{\hat{y}_{k}^{T} s_{k}} .
\end{aligned}
$$


In addition,

$$
\gamma_{i} \frac{\left\|\widehat{y}_{i}\right\|^{2}}{\hat{y}_{i}^{T} s_{i}}=\frac{\left\|\hat{y}_{i}\right\|^{2}}{\left\|\hat{y}_{i}\right\|^{2}+\left\|s_{i}\right\|^{2}} \leq \frac{1}{2}
$$

Therefore, by Remark 1 and the above inequality, the formula (33) is transformed into

$$
\operatorname{tr}\left(B_{k+1}\right) \leq \Lambda \operatorname{tr}\left(B_{0}\right)-\sum_{i=0}^{k} \delta \frac{\left\|B_{i} s_{i}\right\|^{2}}{s_{i}^{T} B_{i} s_{i}}+\sum_{j=1}^{k} \Lambda+\frac{1}{2} \leq \Lambda \operatorname{tr}\left(B_{0}\right)+\Lambda k+\frac{1}{2},
$$

which implies (31). From the positive definiteness of $B_{k+1}$, (32) also holds. The proof is completed.

Lemma 4. Consider $\gamma_{k} \geq s$ and $\delta_{k} \geq q$ for all $k$, where $s$ and $t$ are constants. Then, there exists a positive constant $h$ such that

$$
\prod_{i=0}^{k} \alpha_{i} \geq h^{k}
$$

for all $k$ sufficiently large.

Proof. Utilizing the identity (26) and taking the determinant on both sides of the formula (14) with $\gamma_{k}$ and $\delta_{k}$ computed as in (18) and (16), we have

$$
\begin{aligned}
& \operatorname{det}\left(B_{k+1}\right)=\operatorname{det}\left(\delta_{k} B_{k}\left(I-\frac{s_{k} s_{k}^{T} B_{k}}{s_{k}^{T} B_{k} s_{k}}+\frac{\gamma_{k}}{\delta_{k}} \frac{B_{k}^{-1} \widehat{y}_{k} \widehat{y}_{k}^{T}}{\widehat{y}_{k}^{T} s_{k}}\right)\right) \\
& =\operatorname{det}\left(\delta_{k} B_{k}\right) \operatorname{det}\left(I-s_{k} \frac{\left(B_{k} s_{k}\right)^{T}}{s_{k}^{T} B_{k} s_{k}}+\frac{\gamma_{k}}{\delta_{k}}\left(B_{k}^{-1} \widehat{y}_{k}\right) \frac{\widehat{y}_{k}^{T}}{\widehat{y}_{k}^{T} s_{k}}\right) \\
& =\delta_{k}^{n} \operatorname{det}\left(B_{k}\right) \frac{\gamma_{k}}{\delta_{k}} \frac{\widehat{y}_{k}^{T} s_{k}}{s^{T} B_{k} s_{k}} \\
& =\left(\delta_{k}^{n-1} \gamma_{k} \frac{\widehat{y}_{k}^{T} s_{k}}{s_{k}^{T} B_{k} s_{k}}\right)\left(\delta_{k-1}^{n-1} \gamma_{k-1} \frac{\hat{y}_{k-1}^{T} s_{k-1}}{s_{k-1}^{T} B_{k-1} s_{k-1}}\right) \operatorname{det}\left(B_{k-1}\right) \\
& =\left(\delta_{k}^{n-1} \gamma_{k} \frac{\widehat{y}_{k}^{T} s_{k}}{s_{k}^{T} B_{k} s_{k}}\right)\left(\delta_{k-1}^{n-1} \gamma_{k-1} \frac{\widehat{y}_{k-1}^{T} s_{k-1}}{s_{k-1}^{T} B_{k-1} s_{k-1}}\right), \ldots, \\
& \left(\delta_{0}^{n-1} \gamma_{0} \frac{\hat{y}_{0}^{T} s_{0}}{s_{0}^{T} B_{0} s_{0}}\right) \operatorname{det}\left(B_{0}\right) \\
& =\left(\prod_{i=0}^{k} \delta_{i}^{n-1} \gamma_{i} \frac{\hat{y}_{i}^{T} s_{i}}{s_{i}^{T} B_{i} s_{i}}\right) \operatorname{det}\left(B_{0}\right) \\
& \geq \operatorname{det}\left(B_{0}\right) \prod_{i=0}^{k} q^{n-1} s \frac{1-\sigma}{\alpha_{i}} \\
& =\operatorname{det}\left(B_{0}\right) q^{(n-1)(k+1)} s^{k+1}(1-\sigma)^{k+1} \prod_{i=0}^{k} \frac{1}{\alpha_{i}},
\end{aligned}
$$

where the penultimate inequality follows $s_{i}^{T} B_{i} s_{i}=-\alpha_{i} s_{i}^{T} g_{i}$, $\hat{y}_{i}^{T} s_{i} \geq-(1-\sigma) s_{i}^{T} g_{i}, \gamma_{i} \geq s$, and $\delta_{i} \geq q$ for all $i$. Furthermore, by $\operatorname{det}\left(B_{k+1}\right) \leq(1 / n) \operatorname{tr}\left(B_{k+1}\right)^{n}$ and Lemma 4 , we obtain

$$
\operatorname{det}\left(B_{k+1}\right) \leq\left(\frac{1}{n}\left(\Lambda \operatorname{tr}\left(B_{0}\right)+\Lambda k+\frac{1}{2}\right)\right)^{n} .
$$

Therefore,

$$
\begin{aligned}
\prod_{i=0}^{k} \alpha_{i} & \geq \frac{\operatorname{det}\left(B_{0}\right) q^{(n-1)(k+1)} s^{k+1}(1-\sigma)^{k+1}}{\operatorname{det}\left(B_{k+1}\right)} \\
& \geq \frac{\operatorname{det}\left(B_{0}\right) q^{(n-1)(k+1)} s^{k+1}(1-\sigma)^{k+1}}{\left((1 / n)\left(\Lambda \operatorname{tr}\left(B_{0}\right)+\Lambda k+(1 / 2)\right)\right)^{n}} .
\end{aligned}
$$

Suppose $k$ is sufficiently large, (39) implies (36). The proof is completed.

Theorem 1. If the sequence $\left\{x_{k}\right\}$ is obtained by Algorithm 1, then

$$
\underset{k \rightarrow \infty}{\liminf }\left\|g_{k}\right\|=0
$$

Proof. The proof by contradiction is used to prove (40) holds. Suppose that $\left\|g_{k}\right\|>F>0$. By Yuan-Wei-Lu line search (22) and $f(x)$ bounded below, we obtain

$$
\begin{aligned}
f_{k}-f\left(x_{k}+\alpha_{k} d_{k}\right) & \geq-\xi \alpha_{k} g_{k}^{T} d_{k}-\alpha_{k} \min \left[-\xi_{1} g_{k}^{T} d_{k}, \xi \frac{\alpha_{k}}{2}\left\|d_{k}\right\|^{2}\right] \\
& \geq-\left(\xi-\xi_{1}\right) \alpha_{k} g_{k}^{T} d_{k} .
\end{aligned}
$$

Adding the abovementioned inequalities from $k=0$ to $\infty$ and utilizing Assumption 1 (ii), we have

$$
\sum_{k=0}^{\infty}\left(-s_{k}^{T} g_{k}\right)<\infty
$$

From Assumption 1 (ii) and (42), we have

$$
\begin{aligned}
\infty>\sum_{k=0}^{\infty}\left(-s_{k}^{T} g_{k}\right) & =\sum_{k=0}^{\infty} \frac{1}{\alpha_{k}} s_{k}^{T} B_{k} s_{k} \\
& =\sum_{k=0}^{\infty} \frac{\left\|g_{k}\right\|}{\left\|B_{k} s_{k}\right\|} s_{k}^{T} B_{k} s_{k} \\
& =\sum_{k=0}^{\infty} \frac{s_{k}^{T} B_{k} s_{k}}{\left\|B_{k} s_{k}\right\|}\left\|g_{k}\right\| \frac{\alpha_{k}\left\|g_{k}\right\|}{\left\|B_{k} s_{k}\right\|} \\
& =\sum_{k=0}^{\infty}\left\|g_{k}\right\|^{2} \alpha_{k} \frac{s_{k}^{T} B_{k} s_{k}}{\left\|B_{k} s_{k}\right\|^{2}} \\
& \geq F^{2} \sum_{k=0}^{\infty} \alpha_{k} \frac{s_{k}^{T} B_{k} s_{k}}{\left\|B_{k} s_{k}\right\|^{2}} .
\end{aligned}
$$

Based on this, given a constant $\Phi>0$, there is a positive integer $k_{0}>0$ satisfying 
TABLE 1: The test problems.

\begin{tabular}{|c|c|}
\hline No. & Test problem \\
\hline 1 & Extended Freudenstein and Roth function \\
\hline 2 & Extended trigonometric function \\
\hline 3 & Extended Rosenbrock function \\
\hline 4 & Extended White and Holst function \\
\hline 5 & Extended Beale function \\
\hline 6 & Extended penalty function \\
\hline 7 & Perturbed quadratic function \\
\hline 8 & Raydan 1 function \\
\hline 9 & Raydan 2 function \\
\hline 10 & Diagonal 1 function \\
\hline 11 & Diagonal 2 function \\
\hline 12 & Diagonal 3 function \\
\hline 13 & Hager function \\
\hline 14 & Generalized tridiagonal 1 function \\
\hline 15 & Extended tridiagonal 1 function \\
\hline 16 & Extended three exponential terms function \\
\hline 17 & Generalized tridiagonal 2 function \\
\hline 18 & Diagonal 4 function \\
\hline 19 & Diagonal 5 function \\
\hline 20 & Extended Himmelblau function \\
\hline 21 & Generalized PSC1 function \\
\hline 22 & Extended PSC1 function \\
\hline 23 & Extended Powell function \\
\hline 24 & Extended block diagonal BD1 function \\
\hline 25 & Extended Maratos function \\
\hline 26 & Extended Cliff function \\
\hline 27 & Quadratic diagonal perturbed function \\
\hline 28 & Extended Wood function \\
\hline 29 & Extended Hiebert function \\
\hline 30 & Quadratic function QF1 function \\
\hline 31 & Extended quadratic penalty QP1 function \\
\hline 32 & Extended quadratic penalty QP2 function \\
\hline 33 & A quadratic function QF2 function \\
\hline 34 & Extended EP1 function \\
\hline 35 & Extended tridiagonal 2 function \\
\hline 36 & BDQRTIC function (CUTE) \\
\hline 37 & TRIDIA function (CUTE) \\
\hline 38 & ARWHEAD function (CUTE) \\
\hline 39 & NONDIA function (CUTE) \\
\hline 40 & NONDQUAR function (CUTE) \\
\hline 41 & DQDRTIC function (CUTE) \\
\hline 42 & EG2 function (CUTE) \\
\hline 43 & DIXMAANA function (CUTE) \\
\hline 44 & DIXMAANB function (CUTE) \\
\hline 45 & DIXMAANC function (CUTE) \\
\hline 46 & DIXMAANE function (CUTE) \\
\hline 47 & Partial perturbed quadratic function \\
\hline 48 & Broyden tridiagonal function \\
\hline 49 & Almost perturbed quadratic function \\
\hline 50 & Tridiagonal perturbed quadratic function \\
\hline 51 & EDENSCH function (CUTE) \\
\hline 52 & VARDIM function (CUTE) \\
\hline 53 & STAIRCASE S1 function \\
\hline 54 & LIARWHD function (CUTE) \\
\hline 55 & DIAGONAL 6 function \\
\hline 56 & DIXON3DQ function (CUTE) \\
\hline 57 & DIXMAANF function (CUTE) \\
\hline 58 & DIXMAANG function (CUTE) \\
\hline 59 & DIXMAANH function (CUTE) \\
\hline 60 & DIXMAANI function (CUTE) \\
\hline
\end{tabular}

TABle 1: Continued.

\begin{tabular}{lc}
\hline No. & Test problem \\
\hline 61 & DIXMAANJ function (CUTE) \\
62 & DIXMAANK function (CUTE) \\
63 & DIXMAANL function (CUTE) \\
64 & DIXMAAND function (CUTE) \\
65 & ENGVAL1 function (CUTE) \\
66 & FLETCHCR function (CUTE) \\
67 & COSINE function (CUTE) \\
68 & Extended DENSCHNB function (CUTE) \\
69 & Extended DENSCHNF function (CUTE) \\
70 & SINQUAD function (CUTE) \\
71 & BIGGSB1 function (CUTE) \\
72 & Partial perturbed quadratic PPQ2 function \\
73 & Scaled quadratic SQ1 function \\
74 & Scaled quadratic SQ2 function \\
\hline
\end{tabular}

$$
c\left[\prod_{k=k_{0}+1}^{k_{0}+c} \alpha_{k} \frac{s_{k}^{T} B_{k} s_{k}}{\left\|B_{k} s_{k}\right\|^{2}}\right]^{(1 / c)} \leq \prod_{k=k_{0}+1}^{k_{0}+c} \alpha_{k} \frac{s_{k}^{T} B_{k} s_{k}}{\left\|B_{k} s_{k}\right\|^{2}} \leq \Phi
$$

where $c$ is any positive integer, and the first inequality follows the geometric inequality. Moreover, by Lemma 4, we obtain

$$
\begin{aligned}
{\left[\prod_{k=k_{0}+1}^{k_{0}+c} \alpha_{k}\right]^{(1 / c)} } & \leq \frac{\Phi}{c}\left[\prod_{k=k_{0}+1}^{k_{0}+c} \frac{\left\|B_{k} s_{k}\right\|^{2}}{s_{k}^{T} B_{k} s_{k}}\right]^{(1 / c)} \\
& \leq \frac{\Phi}{c^{2}} \sum_{k=0}^{k_{0}+c} \frac{\left\|B_{k} s_{k}\right\|^{2}}{s_{k}^{T} B_{k} s_{k}} \\
& \leq \frac{\Phi}{c^{2} \delta}\left(\Lambda \operatorname{tr}\left(B_{0}\right)+\Lambda\left(k_{0}+c\right)+\frac{1}{2}\right)
\end{aligned}
$$

Considering $c \longrightarrow \infty$, the above formula and formula (39) are contradictory. Thus, (40) is valid. The proof is completed.

\section{Numerical Results}

In this section, numerical results of Algorithm 1 are reported, and the following methods were compared: (i) MTPSBFGS method $\left(B_{k+1}\right.$ is updated by (17) with $\gamma_{k}$ and $\delta_{k}$ given by (18) and (19)). (ii) SBFGS method ( $B_{k+1}$ is updated by (14) with $\gamma_{k}$ and $\delta_{k}$ given by (11) and (16)).

\subsection{General Unconstrained Optimisation Problems}

Tested problems: a total of 74 test questions, listed in Table 1 and derived from the studies by Bongartz et al. and More et al. [47, 48].

Parameters: Algorithm 1 runs with $\delta=0.2, \delta_{1}=0.15$, $\sigma=0.85, \beta_{k}=10^{-17}$, and $B_{0}=I$.

Dimensionality: the algorithm is tested in the following three dimensions: 300,900 , and 2700 . 


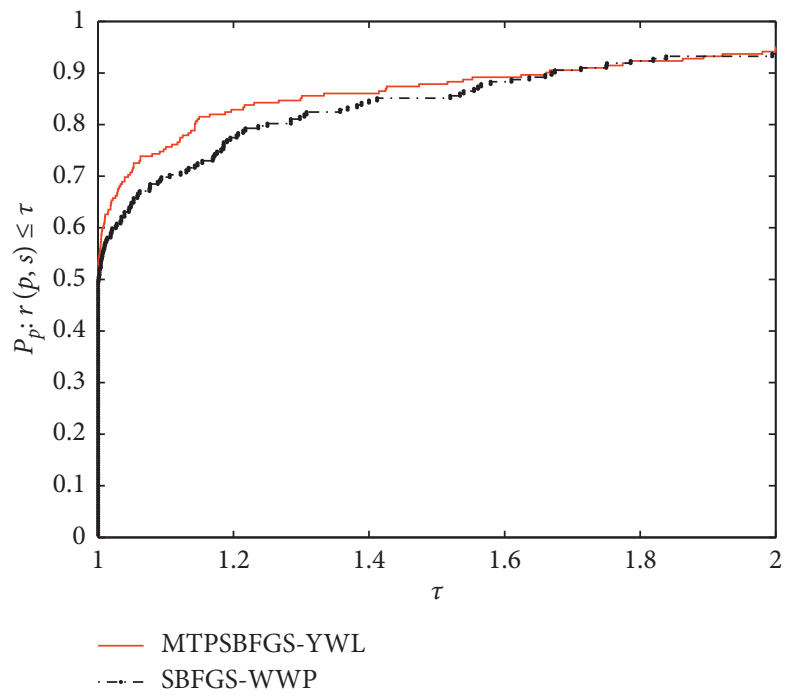

FIgURE 1: Performance profiles of these methods (CPU time).

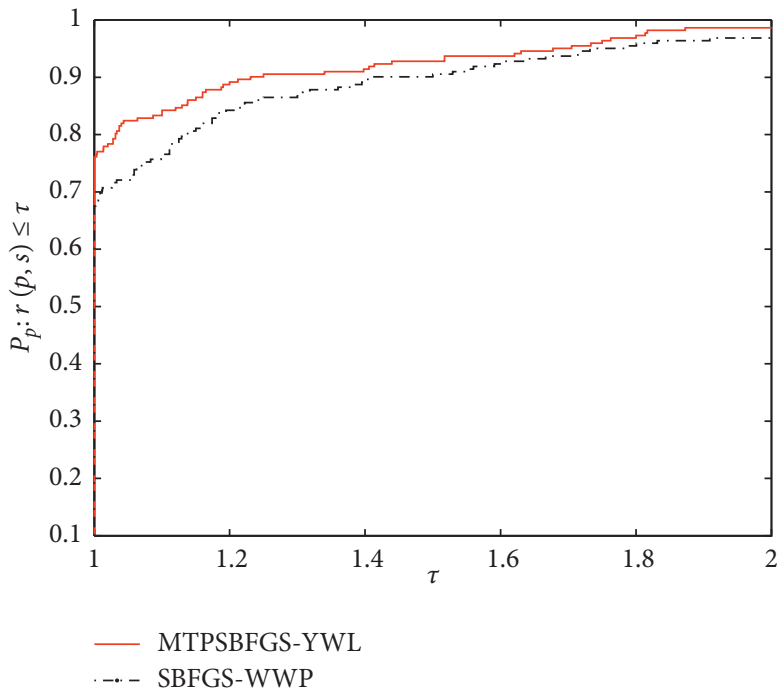

Figure 2: Performance profiles of these methods (NI).

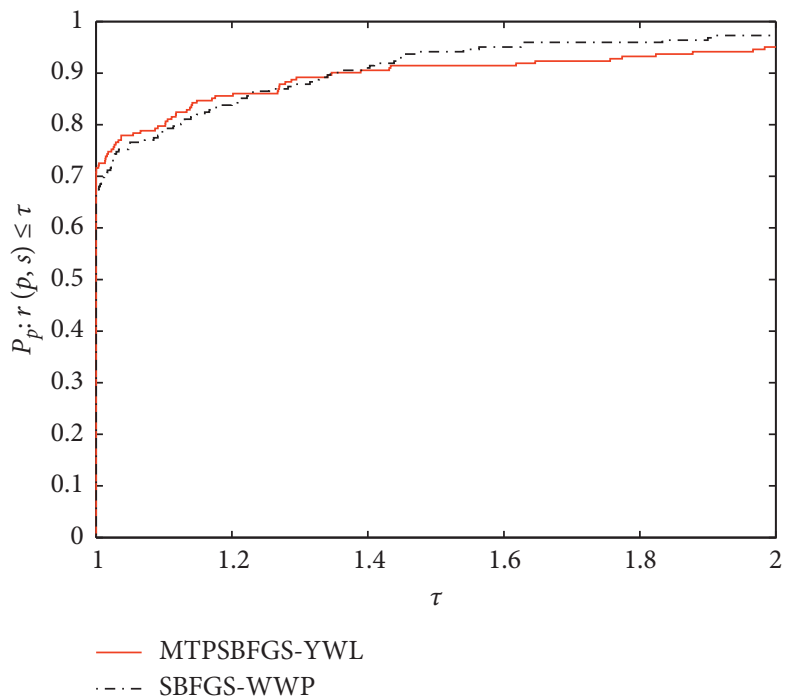

Figure 3: Performance profiles of these methods (NFG). 
TABLE 2: The numerical results for problems 1-17.

\begin{tabular}{|c|c|c|c|c|c|c|c|}
\hline \multicolumn{5}{|c|}{ MTPSBFGS-YWL } & \multicolumn{3}{|c|}{ SBFGS-WWP } \\
\hline No. & Dim & NI & NFG & CPU time & $\mathrm{NI}$ & NFG & CPU time \\
\hline 1 & 300 & 29 & 63 & 0.3125 & 25 & 57 & 0.3125 \\
\hline 1 & 900 & 24 & 56 & 5.859375 & 23 & 54 & 5.96875 \\
\hline 1 & 2700 & 22 & 48 & 86.9375 & 29 & 67 & 135.6875 \\
\hline 2 & 300 & 50 & 114 & 0.59375 & 46 & 102 & 0.5 \\
\hline 2 & 900 & 51 & 114 & 12.890625 & 50 & 112 & 13.515625 \\
\hline 2 & 2700 & 53 & 120 & 217.90625 & 53 & 122 & 254.75 \\
\hline 3 & 300 & 50 & 137 & 0.515625 & 47 & 120 & 0.5 \\
\hline 3 & 900 & 75 & 215 & 17.59375 & 63 & 167 & 16.75 \\
\hline 3 & 2700 & 43 & 135 & 184.5625 & 60 & 166 & 280.484375 \\
\hline 4 & 300 & 104 & 370 & 1.25 & 62 & 176 & 0.75 \\
\hline 4 & 900 & 87 & 258 & 22.453125 & 38 & 109 & 9.1875 \\
\hline 4 & 2700 & 67 & 179 & 308.3125 & 50 & 149 & 236.984375 \\
\hline 5 & 300 & 18 & 48 & 0.21875 & 22 & 58 & 0.296875 \\
\hline 5 & 900 & 18 & 45 & 4.5625 & 20 & 46 & 5.171875 \\
\hline 5 & 2700 & 20 & 45 & 90.25 & 23 & 58 & 106.984375 \\
\hline 6 & 300 & 68 & 152 & 0.828125 & 68 & 152 & 0.796875 \\
\hline 6 & 900 & 69 & 158 & 18.375 & 69 & 158 & 18.40625 \\
\hline 6 & 2700 & 85 & 192 & 405.84375 & 85 & 192 & 410.765625 \\
\hline 7 & 300 & 76 & 154 & 0.8125 & 76 & 154 & 0.875 \\
\hline 7 & 900 & 133 & 268 & 36.6875 & 133 & 268 & 36.84375 \\
\hline 7 & 2700 & 232 & 466 & 1158.734375 & 231 & 464 & 1151.625 \\
\hline 8 & 300 & 22 & 49 & 0.203125 & 26 & 54 & 0.265625 \\
\hline 8 & 900 & 25 & 55 & 6.515625 & 25 & 55 & 6.421875 \\
\hline 8 & 2700 & 25 & 55 & 118.640625 & 25 & 55 & 116.859375 \\
\hline 9 & 300 & 7 & 16 & 0.0625 & 12 & 26 & 0.109375 \\
\hline 9 & 900 & 7 & 16 & 1.546875 & 12 & 26 & 2.84375 \\
\hline 9 & 2700 & 8 & 18 & 31.96875 & 12 & 26 & 49.046875 \\
\hline 10 & 300 & 2 & 9 & 0 & 2 & 9 & 0 \\
\hline 10 & 900 & 2 & 9 & 0.0625 & 2 & 9 & 0.0625 \\
\hline 10 & 2700 & 2 & 9 & 0.25 & 2 & 9 & 0.25 \\
\hline 11 & 300 & 75 & 194 & 0.921875 & 46 & 94 & 0.59375 \\
\hline 11 & 900 & 95 & 272 & 26.359375 & 66 & 134 & 18.484375 \\
\hline 11 & 2700 & 6 & 20 & 19.875 & 97 & 196 & 472.390625 \\
\hline 12 & 300 & 11 & 24 & 0.125 & 11 & 24 & 0.109375 \\
\hline 12 & 900 & 13 & 28 & 3.453125 & 13 & 28 & 3.296875 \\
\hline 12 & 2700 & 13 & 28 & 60.34375 & 13 & 28 & 58.40625 \\
\hline 13 & 300 & 11 & 25 & 0.125 & 10 & 23 & 0.125 \\
\hline 13 & 900 & 8 & 23 & 1.921875 & 10 & 26 & 2.46875 \\
\hline 13 & 2700 & 19 & 94 & 88.65625 & 19 & 96 & 88.71875 \\
\hline 14 & 300 & 8 & 20 & 0.125 & 8 & 20 & 0.15625 \\
\hline 14 & 900 & 7 & 18 & 1.703125 & 7 & 18 & 1.703125 \\
\hline 14 & 2700 & 7 & 18 & 27.6875 & 7 & 18 & 27.421875 \\
\hline 15 & 300 & 19 & 43 & 0.359375 & 22 & 49 & 0.40625 \\
\hline 15 & 900 & 25 & 57 & 7.125 & 39 & 82 & 11.078125 \\
\hline 15 & 2700 & 25 & 55 & 119.40625 & 41 & 86 & 198.203125 \\
\hline 16 & 300 & 9 & 21 & 0.109375 & 10 & 23 & 0.0625 \\
\hline 16 & 900 & 8 & 18 & 1.921875 & 9 & 21 & 2.15625 \\
\hline 16 & 2700 & 11 & 24 & 49.359375 & 10 & 22 & 43.046875 \\
\hline 17 & 300 & 33 & 73 & 0.421875 & 32 & 75 & 0.390625 \\
\hline 17 & 900 & 23 & 49 & 5.984375 & 23 & 49 & 5.8125 \\
\hline 17 & 2700 & 25 & 53 & 115.890625 & 25 & 53 & 112.984375 \\
\hline
\end{tabular}

Himmelblau stop rule [49]: if $\left|f\left(x_{k}\right)\right|>e_{1}$, then set $S_{1}=$ $\left|f\left(x_{k}\right)-f\left(x_{k+1}\right)\right|$ or $\left(\left|f\left(x_{k}\right)-f\left(x_{k+1}\right)\right| /\left|f\left(x_{k}\right)\right|\right)$. The iterations are stopped if $\|g(x)\|<\varepsilon$ or $S_{1}<e_{2}$ holds, where $e_{1}=e_{2}=10^{-5}$ and $\epsilon=10^{-6}$.
Experiment environment: all programs are written in MATLAB R2014a and run on a PC with an Inter(R) Core(TM) i5-4210U CPU at $1.70 \mathrm{GHz}, 8.00 \mathrm{~GB}$ of RAM, and the Windows 10 operating system. 
TABLE 3: The numerical results for problems $18-34$.

\begin{tabular}{|c|c|c|c|c|c|c|c|}
\hline \multicolumn{5}{|c|}{ MTPSBFGS-YWL } & \multicolumn{3}{|c|}{ SBFGS-WWP } \\
\hline No. & Dim & NI & NFG & CPU time & NI & NFG & CPU time \\
\hline 18 & 300 & 3 & 10 & 0 & 3 & 10 & 0 \\
\hline 18 & 900 & 3 & 10 & 0.53125 & 3 & 10 & 0.5 \\
\hline 18 & 2700 & 3 & 10 & 9.09375 & 3 & 10 & 8.75 \\
\hline 19 & 300 & 3 & 10 & 0 & 3 & 10 & 0.0625 \\
\hline 19 & 900 & 3 & 10 & 0.53125 & 3 & 10 & 0.484375 \\
\hline 19 & 2700 & 3 & 10 & 8.578125 & 3 & 10 & 8.421875 \\
\hline 20 & 300 & 33 & 71 & 0.34375 & 32 & 69 & 0.359375 \\
\hline 20 & 900 & 12 & 31 & 2.234375 & 12 & 31 & 2.171875 \\
\hline 20 & 2700 & 12 & 35 & 44.546875 & 43 & 93 & 191.3125 \\
\hline 21 & 300 & 25 & 56 & 0.28125 & 34 & 74 & 0.390625 \\
\hline 21 & 900 & 25 & 56 & 6.703125 & 35 & 76 & 9.265625 \\
\hline 21 & 2700 & 26 & 58 & 124.484375 & 36 & 78 & 170.34375 \\
\hline 22 & 300 & 8 & 30 & 0.109375 & 8 & 31 & 0.125 \\
\hline 22 & 900 & 8 & 31 & 1.90625 & 8 & 31 & 2 \\
\hline 22 & 2700 & 8 & 31 & 33.515625 & 8 & 31 & 33.359375 \\
\hline 23 & 300 & 34 & 85 & 0.359375 & 52 & 109 & 0.5625 \\
\hline 23 & 900 & 39 & 89 & 10.65625 & 51 & 117 & 13.6875 \\
\hline 23 & 2700 & 47 & 111 & 233.65625 & 47 & 112 & 223.71875 \\
\hline 24 & 300 & 33 & 162 & 0.296875 & 29 & 145 & 0.234375 \\
\hline 24 & 900 & 14 & 111 & 1.03125 & 37 & 171 & 6.890625 \\
\hline 24 & 2700 & 14 & 111 & 15.34375 & 15 & 114 & 24.234375 \\
\hline 25 & 300 & 90 & 262 & 1.0625 & 127 & 348 & 1.5 \\
\hline 25 & 900 & 123 & 346 & 33.765625 & 88 & 257 & 23.703125 \\
\hline 25 & 2700 & 56 & 139 & 270.703125 & 97 & 284 & 463.609375 \\
\hline 26 & 300 & 56 & 134 & 0.640625 & 56 & 134 & 0.609375 \\
\hline 26 & 900 & 65 & 152 & 17.359375 & 65 & 152 & 17.296875 \\
\hline 26 & 2700 & 61 & 146 & 292.546875 & 61 & 146 & 287.25 \\
\hline 27 & 300 & 6 & 16 & 0.0625 & 6 & 16 & 0 \\
\hline 27 & 900 & 11 & 31 & 2.75 & 11 & 31 & 2.65625 \\
\hline 27 & 2700 & 16 & 45 & 75.3125 & 17 & 44 & 79.671875 \\
\hline 28 & 300 & 28 & 63 & 0.3125 & 27 & 61 & 0.296875 \\
\hline 28 & 900 & 28 & 63 & 7.34375 & 27 & 62 & 6.921875 \\
\hline 28 & 2700 & 25 & 61 & 119.5 & 25 & 61 & 117.265625 \\
\hline 29 & 300 & 4 & 17 & 0.0625 & 4 & 15 & 0.046875 \\
\hline 29 & 900 & 4 & 17 & 0.6875 & 4 & 17 & 0.828125 \\
\hline 29 & 2700 & 4 & 17 & 9.484375 & 4 & 17 & 9.53125 \\
\hline 30 & 300 & 93 & 188 & 1.203125 & 93 & 188 & 1.046875 \\
\hline 30 & 900 & 164 & 330 & 45.96875 & 166 & 334 & 46.890625 \\
\hline 30 & 2700 & 295 & 592 & 1476.90625 & 294 & 590 & 1474.96875 \\
\hline 31 & 300 & 23 & 52 & 0.21875 & 23 & 52 & 0.21875 \\
\hline 31 & 900 & 27 & 62 & 6.828125 & 27 & 62 & 6.84375 \\
\hline 31 & 2700 & 28 & 64 & 126.734375 & 28 & 64 & 126.421875 \\
\hline 32 & 300 & 22 & 46 & 0.28125 & 22 & 46 & 0.25 \\
\hline 32 & 900 & 20 & 44 & 5.171875 & 20 & 44 & 5.03125 \\
\hline 32 & 2700 & 47 & 98 & 219.515625 & 47 & 98 & 218.78125 \\
\hline 33 & 300 & 5 & 11 & 0.0625 & 5 & 11 & 0.0625 \\
\hline 33 & 900 & 4 & 9 & 0.625 & 4 & 9 & 0.59375 \\
\hline 33 & 2700 & 3 & 7 & 7.421875 & 3 & 7 & 7.34375 \\
\hline 34 & 300 & 3 & 7 & 0.0625 & 3 & 7 & 0 \\
\hline 34 & 900 & 3 & 7 & 0.53125 & 3 & 7 & 0.5 \\
\hline 34 & 2700 & 4 & 8 & 13.203125 & 4 & 8 & 13.234375 \\
\hline
\end{tabular}

Symbol representation: No.: the test problem number. CPU time: the CPU time in seconds. NI: the number of iterations. NFG: the total number of function and gradient evaluations.
Image description: Figures 1-3 show the profiles for CPU time, NI, and NFG, and Tables $2-6$ provide the detail numerical results. From these figures and tables, it is obvious that the MTPSBFGS method possesses 
TABLE 4: The numerical results for problems 35-51.

\begin{tabular}{|c|c|c|c|c|c|c|c|}
\hline \multirow[b]{2}{*}{ No. } & \multicolumn{3}{|c|}{ MTPSBFGS-YWL } & \multirow[b]{2}{*}{ CPU time } & \multicolumn{3}{|c|}{ SBFGS-WWP } \\
\hline & Dim & $\mathrm{NI}$ & NFG & & NI & NFG & CPU time \\
\hline 35 & 300 & 4 & 8 & 0 & 4 & 8 & 0 \\
\hline 35 & 900 & 7 & 14 & 1.59375 & 7 & 14 & 1.59375 \\
\hline 35 & 2700 & 11 & 22 & 46.875 & 11 & 22 & 46.40625 \\
\hline 36 & 300 & 24 & 59 & 0.359375 & 26 & 64 & 0.46875 \\
\hline 36 & 900 & 23 & 66 & 6.359375 & 20 & 58 & 5.59375 \\
\hline 36 & 2700 & 18 & 49 & 82.359375 & 19 & 46 & 85.5625 \\
\hline 37 & 300 & 136 & 275 & 1.65625 & 136 & 275 & 1.6875 \\
\hline 37 & 900 & 235 & 473 & 67.40625 & 235 & 473 & 67.359375 \\
\hline 37 & 2700 & 441 & 886 & 2240.015625 & 442 & 888 & 2234.09375 \\
\hline 38 & 300 & 12 & 27 & 0.125 & 12 & 27 & 0.0625 \\
\hline 38 & 900 & 12 & 26 & 2.8125 & 12 & 26 & 2.921875 \\
\hline 38 & 2700 & 15 & 33 & 64.90625 & 15 & 33 & 64.65625 \\
\hline 39 & 300 & 37 & 80 & 0.40625 & 37 & 80 & 0.46875 \\
\hline 39 & 900 & 43 & 89 & 10.890625 & 43 & 91 & 11.265625 \\
\hline 39 & 2700 & 26 & 52 & 116.703125 & 26 & 52 & 116.078125 \\
\hline 40 & 300 & 532 & 1329 & 7.40625 & 958 & 1925 & 12.96875 \\
\hline 40 & 900 & 546 & 1364 & 150.921875 & 1000 & 2008 & 274.5625 \\
\hline 40 & 2700 & 644 & 1605 & 3124.65625 & 1000 & 2014 & 4810.296875 \\
\hline 41 & 300 & 18 & 41 & 0.1875 & 20 & 45 & 0.1875 \\
\hline 41 & 900 & 19 & 43 & 4.828125 & 19 & 43 & 4.734375 \\
\hline 41 & 2700 & 19 & 43 & 85.0625 & 19 & 43 & 84.875 \\
\hline 42 & 300 & 19 & 65 & 0.15625 & 16 & 57 & 0.078125 \\
\hline 42 & 900 & 4 & 21 & 0.046875 & 4 & 21 & 0.046875 \\
\hline 42 & 2700 & 4 & 21 & 0.578125 & 4 & 21 & 0.515625 \\
\hline 43 & 300 & 22 & 48 & 0.265625 & 25 & 54 & 0.234375 \\
\hline 43 & 900 & 23 & 50 & 5.84375 & 27 & 58 & 6.984375 \\
\hline 43 & 2700 & 25 & 54 & 114.328125 & 29 & 62 & 133.78125 \\
\hline 44 & 300 & 38 & 80 & 0.453125 & 39 & 82 & 0.546875 \\
\hline 44 & 900 & 36 & 76 & 9.703125 & 43 & 90 & 11.5 \\
\hline 44 & 2700 & 39 & 82 & 180.453125 & 46 & 96 & 212.125 \\
\hline 45 & 300 & 17 & 40 & 0.171875 & 18 & 42 & 0.28125 \\
\hline 45 & 900 & 17 & 40 & 4.265625 & 18 & 42 & 4.65625 \\
\hline 45 & 2700 & 18 & 42 & 80.171875 & 19 & 44 & 85.09375 \\
\hline 46 & 300 & 104 & 255 & 1.5625 & 60 & 126 & 0.875 \\
\hline 46 & 900 & 141 & 354 & 41.203125 & 87 & 180 & 24.796875 \\
\hline 46 & 2700 & 164 & 418 & 829.3125 & 116 & 238 & 586.296875 \\
\hline 47 & 300 & 37 & 80 & 0.734375 & 36 & 78 & 0.71875 \\
\hline 47 & 900 & 44 & 95 & 14.640625 & 44 & 95 & 14.78125 \\
\hline 47 & 2700 & 13 & 31 & 64.21875 & 13 & 31 & 63.9375 \\
\hline 48 & 300 & 26 & 52 & 0.296875 & 26 & 52 & 0.3125 \\
\hline 48 & 900 & 48 & 96 & 12.890625 & 48 & 96 & 12.890625 \\
\hline 48 & 2700 & 22 & 47 & 99.546875 & 22 & 47 & 99.96875 \\
\hline 49 & 300 & 76 & 154 & 0.953125 & 76 & 154 & 0.859375 \\
\hline 49 & 900 & 133 & 268 & 37.203125 & 134 & 270 & 37.59375 \\
\hline 49 & 2700 & 232 & 466 & 1161.109375 & 234 & 470 & 1176.921875 \\
\hline 50 & 300 & 76 & 154 & 1.234375 & 75 & 152 & 1.328125 \\
\hline 50 & 900 & 132 & 266 & 38.859375 & 132 & 266 & 38.53125 \\
\hline 50 & 2700 & 231 & 464 & 1165.1875 & 232 & 466 & 1170 \\
\hline 51 & 300 & 23 & 48 & 0.296875 & 23 & 48 & 0.3125 \\
\hline 51 & 900 & 23 & 48 & 5.890625 & 23 & 48 & 5.859375 \\
\hline 51 & 2700 & 23 & 48 & 102.484375 & 23 & 48 & 103.453125 \\
\hline
\end{tabular}

better numerical performance between these two methods, that is, the proposed modified scaled BFGS method is reasonable and feasible. The specific reasons for good performance are stated as follows. The parameter scaling the first two terms of the standard BFGS update is determined to cluster the eigenvalues of 
TABLE 5: The numerical results for problems 52-68.

\begin{tabular}{|c|c|c|c|c|c|c|c|}
\hline \multicolumn{5}{|c|}{ MTPSBFGS-YWL } & \multicolumn{3}{|c|}{ SBFGS-WWP } \\
\hline No. & Dim & $\mathrm{NI}$ & NFG & CPU time & NI & NFG & CPU time \\
\hline 52 & 300 & 87 & 200 & 0.875 & 87 & 200 & 1.0625 \\
\hline 52 & 900 & 103 & 236 & 27.6875 & 103 & 236 & 27.875 \\
\hline 52 & 2700 & 118 & 270 & 566.65625 & 118 & 270 & 567.828125 \\
\hline 53 & 300 & 938 & 2375 & 12.09375 & 388 & 778 & 4.671875 \\
\hline 53 & 900 & 1000 & 2537 & 282.375 & 1000 & 2002 & 280.203125 \\
\hline 53 & 2700 & 1000 & 2539 & 5006.875 & 1000 & 2002 & 5001.984375 \\
\hline 54 & 300 & 31 & 72 & 0.328125 & 35 & 88 & 0.390625 \\
\hline 54 & 900 & 42 & 107 & 10.8125 & 24 & 65 & 6.09375 \\
\hline 54 & 2700 & 23 & 67 & 102.75 & 27 & 69 & 121.75 \\
\hline 55 & 300 & 9 & 20 & 0.125 & 18 & 38 & 0.265625 \\
\hline 55 & 900 & 10 & 22 & 2.234375 & 20 & 42 & 4.921875 \\
\hline 55 & 2700 & 11 & 24 & 46.3125 & 21 & 44 & 92.390625 \\
\hline 56 & 300 & 1000 & 2607 & 13.03125 & 375 & 750 & 4.5625 \\
\hline 56 & 900 & 1000 & 2540 & 268.3125 & 1000 & 2000 & 277.515625 \\
\hline 56 & 2700 & 1000 & 2539 & 4518.96875 & 1000 & 2000 & 4637.546875 \\
\hline 57 & 300 & 107 & 260 & 1.578125 & 59 & 124 & 0.796875 \\
\hline 57 & 900 & 99 & 236 & 28.140625 & 85 & 176 & 24.15625 \\
\hline 57 & 2700 & 63 & 149 & 313.140625 & 105 & 216 & 522.734375 \\
\hline 58 & 300 & 97 & 233 & 1.3125 & 69 & 144 & 0.890625 \\
\hline 58 & 900 & 120 & 292 & 33.84375 & 99 & 204 & 27.828125 \\
\hline 58 & 2700 & 144 & 344 & 717.328125 & 117 & 240 & 583.078125 \\
\hline 59 & 300 & 24 & 73 & 0.25 & 76 & 169 & 0.890625 \\
\hline 59 & 900 & 27 & 72 & 7.296875 & 27 & 71 & 7.296875 \\
\hline 59 & 2700 & 31 & 78 & 148.625 & 79 & 173 & 389.4375 \\
\hline 60 & 300 & 109 & 260 & 1.484375 & 60 & 126 & 0.796875 \\
\hline 60 & 900 & 132 & 338 & 37.484375 & 87 & 180 & 24.359375 \\
\hline 60 & 2700 & 176 & 434 & 882.109375 & 116 & 238 & 581.953125 \\
\hline 61 & 300 & 104 & 246 & 1.53125 & 59 & 124 & 0.796875 \\
\hline 61 & 900 & 96 & 225 & 26.609375 & 85 & 176 & 24.1875 \\
\hline 61 & 2700 & 66 & 154 & 324.140625 & 105 & 216 & 521.9375 \\
\hline 62 & 300 & 104 & 259 & 1.390625 & 61 & 146 & 0.8125 \\
\hline 62 & 900 & 89 & 232 & 25.265625 & 99 & 220 & 27.625 \\
\hline 62 & 2700 & 126 & 314 & 623.578125 & 105 & 226 & 521.1875 \\
\hline 63 & 300 & 143 & 362 & 2.046875 & 147 & 308 & 2.046875 \\
\hline 63 & 900 & 97 & 259 & 27.59375 & 173 & 365 & 49.28125 \\
\hline 63 & 2700 & 186 & 465 & 933.5 & 199 & 422 & 1005.09375 \\
\hline 64 & 300 & 40 & 88 & 0.53125 & 45 & 98 & 0.578125 \\
\hline 64 & 900 & 28 & 62 & 7.078125 & 32 & 70 & 8.296875 \\
\hline 64 & 2700 & 30 & 66 & 135.65625 & 33 & 72 & 150.015625 \\
\hline 65 & 300 & 22 & 48 & 0.359375 & 22 & 48 & 0.4375 \\
\hline 65 & 900 & 19 & 45 & 5.046875 & 19 & 45 & 5.1875 \\
\hline 65 & 2700 & 18 & 40 & 79.125 & 18 & 40 & 80.765625 \\
\hline 66 & 300 & 611 & 1233 & 11.171875 & 618 & 1238 & 11.6875 \\
\hline 66 & 900 & 1000 & 2003 & 284.8125 & 1000 & 2002 & 286.65625 \\
\hline 66 & 2700 & 1000 & 2003 & 4583.015625 & 1000 & 2002 & 4587.296875 \\
\hline 67 & 300 & 6 & 21 & 0 & 6 & 21 & 0 \\
\hline 67 & 900 & 12 & 33 & 0.25 & 12 & 33 & 0.21875 \\
\hline 67 & 2700 & 10 & 29 & 1.203125 & 13 & 59 & 1.421875 \\
\hline 68 & 300 & 24 & 50 & 0.234375 & 29 & 60 & 0.328125 \\
\hline 68 & 900 & 25 & 52 & 6.453125 & 31 & 64 & 8.375 \\
\hline 68 & 2700 & 27 & 56 & 126.4375 & 33 & 68 & 156.328125 \\
\hline
\end{tabular}

this matrix, and the parameter scaling the third term is determined to reduce its large eigenvalues, thus obtaining a better distribution of them.
4.2. Muskingum Model in Engineering Problems. In this subsection, we present the Muskingum model, and it has the following form: 
TABLE 6: The numerical results for problems 69-74.

\begin{tabular}{|c|c|c|c|c|c|c|c|}
\hline \multicolumn{5}{|c|}{ MTPSBFGS-YWL } & \multicolumn{3}{|c|}{ SBFGS-WWP } \\
\hline No. & Dim & $\mathrm{NI}$ & NFG & CPU time & $\mathrm{NI}$ & NFG & CPU time \\
\hline 69 & 300 & 26 & 56 & 0.265625 & 25 & 54 & 0.21875 \\
\hline 69 & 900 & 28 & 60 & 7.546875 & 25 & 54 & 6.734375 \\
\hline 69 & 2700 & 29 & 62 & 136.421875 & 25 & 54 & 119.25 \\
\hline 70 & 300 & 31 & 84 & 0.5 & 30 & 90 & 0.4375 \\
\hline 70 & 900 & 40 & 103 & 11 & 32 & 88 & 8.46875 \\
\hline 70 & 2700 & 33 & 91 & 146.765625 & 53 & 122 & 245.71875 \\
\hline 71 & 300 & 342 & 894 & 4.171875 & 190 & 381 & 2.203125 \\
\hline 71 & 900 & 1000 & 2572 & 617.34375 & 534 & 1069 & 145.265625 \\
\hline 71 & 2700 & 1000 & 2591 & 7501.1875 & 1000 & 2001 & 4617.703125 \\
\hline 72 & 300 & 124 & 313 & 2.53125 & 109 & 284 & 2.265625 \\
\hline 72 & 900 & 283 & 762 & 94.0625 & 311 & 781 & 107.890625 \\
\hline 72 & 2700 & 843 & 2128 & 4629.25 & 871 & 2163 & 4782.0625 \\
\hline 73 & 300 & 95 & 192 & 1.0625 & 95 & 192 & 1.125 \\
\hline 73 & 900 & 168 & 338 & 46.984375 & 169 & 340 & 46.765625 \\
\hline 73 & 2700 & 296 & 594 & 1477.046875 & 292 & 586 & 1462.71875 \\
\hline 74 & 300 & 37 & 76 & 0.375 & 36 & 74 & 0.34375 \\
\hline 74 & 900 & 50 & 102 & 13.40625 & 50 & 102 & 13.484375 \\
\hline 74 & 2700 & 81 & 164 & 396.390625 & 81 & 164 & 399.5625 \\
\hline
\end{tabular}

TABLE 7: Results of the three algorithms.

\begin{tabular}{lccr}
\hline Algorithm & $x_{1}$ & $x_{2}$ & $x_{3}$ \\
\hline BFGS [52] & 10.8156 & 0.9826 & 1.0219 \\
HIWO [50] & 13.2813 & 0.8001 & 0.9933 \\
MTPSBFGS & 11.1849 & 1.0000 & 0.9996 \\
\hline
\end{tabular}

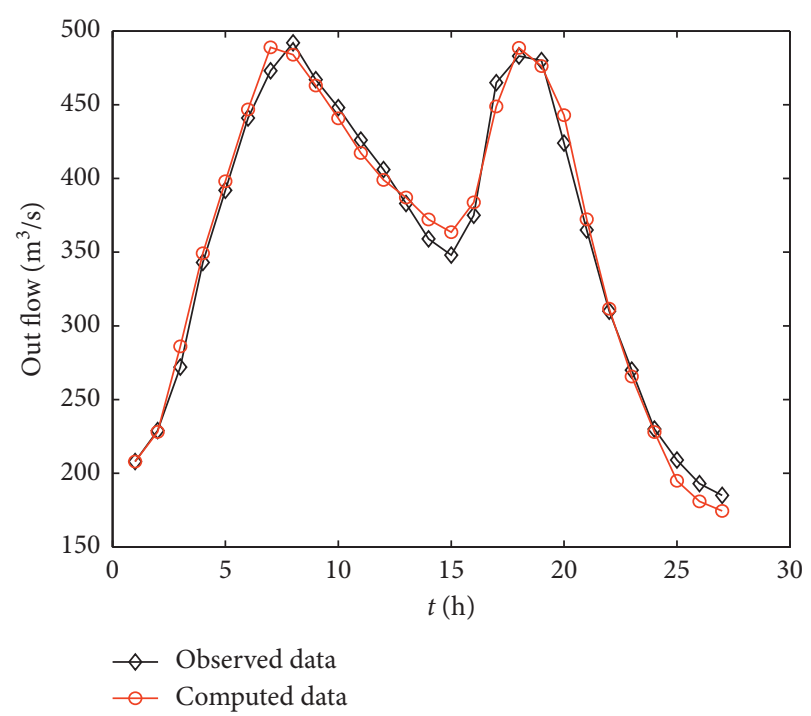

FIgURE 4: Performance of Algorithm 1 in 1960.

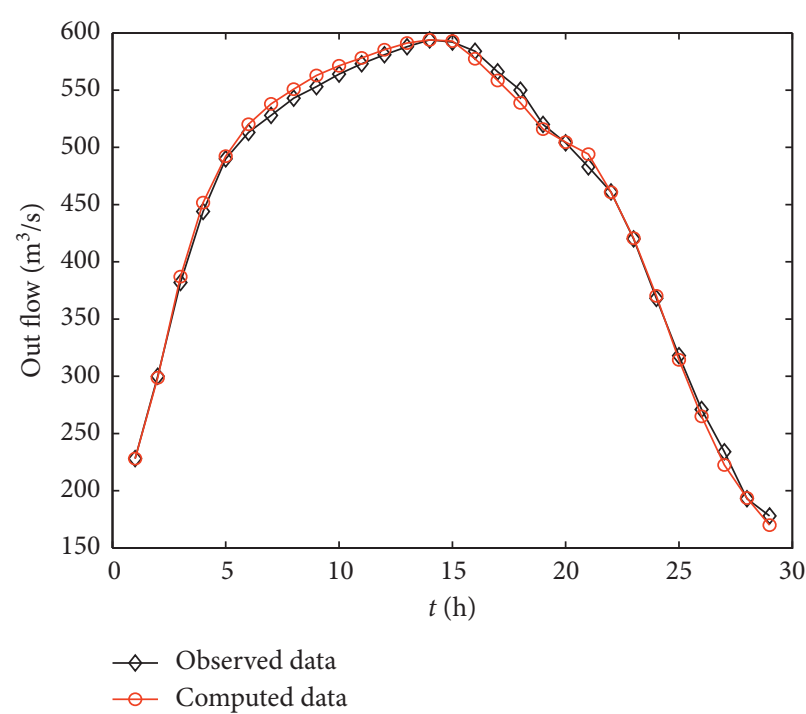

FIgURe 5: Performance of Algorithm 1 in 1961.

Muskingum model [50]:

$\min f\left(x_{1}, x_{2}, x_{3}\right)=\sum_{i=1}^{n-1}\left(\left(1-\frac{\Delta t}{6}\right) x_{1}\left(x_{2} I_{i+1}+\left(1-x_{2}\right) Q_{i+1}\right)^{x_{3}}-\left(1-\frac{\Delta t}{6}\right) x_{1}\left(x_{2} I_{i}+\left(1-x_{2}\right) Q_{i}\right)^{x_{3}}-\frac{\Delta t}{2}\left(I_{i}-Q_{i}\right)+\frac{\Delta t}{2}\left(1-\frac{\Delta t}{3}\right)\left(I_{i+1}-Q_{i+1}\right)\right)^{2}$, 


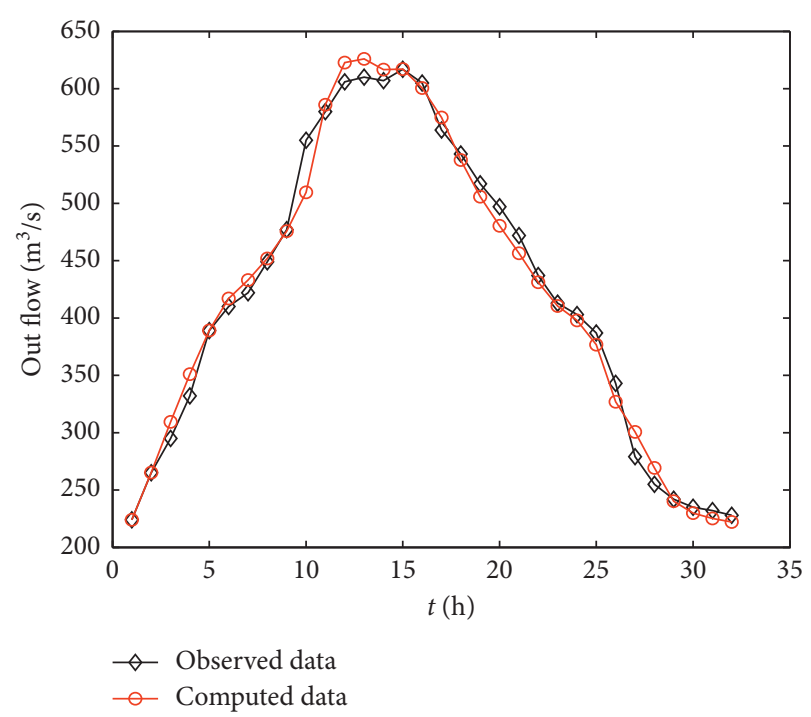

FIgURe 6: Performance of Algorithm 1 in 1964.

whose symbolic representation is as follows: $x_{1}$ is the storage time constant, $x_{2}$ is the weight coefficient, $x_{3}$ is an extra parameter, $I_{i}$ is the observed inflow discharge, $Q_{i}$ is the observed outflow discharge, $n$ is the total time, and $\Delta t$ is the time step at time $t_{i}(i=1,2, \ldots, n)$.

The observed data of the experiment are obtained from the process of flood runoff from Chenggouwan and Linqing of Nanyunhe in the Haihe Basin, Tianjin, China. Select the initial point $x=[0,1,1]^{T}$ and the time step $\Delta t=12(h)$. The concrete values of $I_{i}$ and $Q_{i}$ for the years 1960, 1961, and 1964 are listed in [51]. The test results are presented in Table 7.

Figures 4-6 and Table 7 imply the following three conclusions: (i) based on the Muskingum model, the efficiency of the MTPSBFGS method is wonderful, and numerical performance of these three algorithms is fantastic. (ii) Compared to other similar methods, the final points $\left(x_{1}\right.$, $x_{2}$, and $x_{3}$ ) of the MTPSBFGS method are competitive. (iii) Due to the endpoints of these three methods being different, the Muskingum model may have more approximation optimum points.

\section{Conclusion}

A modified two parameter scaled BFGS method and the Yuan-Wei-Lu line search technology are introduced in this paper. By scaling the first two terms and the third term of the standard BFGS method with different positive parameters, a new two parameter scaled BFGS method is proposed. In this method, the new value of $y_{k}$ is given to guarantee better properties of the new scaled BFGS method. With Yuan-Wei$\mathrm{Lu}$ line search, the proposed BFGS method is globally convergent. Numerical results indicate that the modified two parameter scaled BFGS method outperforms the standard BFGS method and even the same type of the BFGS method. As for the longer-term work, there are several points to consider: (1) are there some new values of $\gamma_{k}, \delta_{k}$, and $y_{k}$ that make the BFGS method based on the update formula (17) perform better? (2) Whether the new scaled method combined with other line search have also great theoretical results. (3) Some new engineering problems based on the BFGS-type method are worth studying.

\section{Data Availability}

The data used to support this study are included within this article.

\section{Conflicts of Interest}

The authors declare that there are no conflicts of interest regarding the publication of this paper.

\section{Acknowledgments}

This work was supported by the National Natural Science Foundation of China (Grant no. 11661009), the High Level Innovation Teams and Excellent Scholars Program in Guangxi Institutions of Higher Education (Grant no. (2019)52), the Guangxi Natural Science Key Fund (Grant no. 2017GXNSFDA198046), and the Guangxi Natural Science Foundation (Grant no. 2020GXNSFAA159069).

\section{References}

[1] R. H. Byrd, S. L. Hansen, J. Nocedal, and Y. Singer, "A stochastic quasi-Newton method for large-scale optimization," SIAM Journal on Optimization, vol. 26, no. 2, pp. 1008-1031, 2016.

[2] A. S. Lewis and M. L. Overton, "Nonsmooth optimization via quasi-Newton methods," Mathematical Programming, vol. 141, no. 1-2, pp. 135-163, 2013.

[3] M. S. Salim and A. I. Ahmed, "A family of Quasi-Newton methods for unconstrained optimization problems," Optimization, vol. 67, no. 10, pp. 1717-1727, 2018.

[4] Z. Wei, G. Li, and L. Qi, "New quasi-Newton methods for unconstrained optimization problems," Applied Mathematics and Computation, vol. 175, no. 2, pp. 1156-1188, 2006.

[5] Z. Wei, G. Yu, G. Yuan, and Z. Lian, "The superlinear convergence of a modified BFGS-type method for unconstrained optimization," Computational Optimization and Applications, vol. 29, no. 3, pp. 315-332, 2004.

[6] G. Yuan, Z. Sheng, B. Wang, W. Hu, and C. Li, "The global convergence of a modified BFGS method for nonconvex functions," Journal of Computational and Applied Mathematics, vol. 327, pp. 274-294, 2018.

[7] W. Zhou and L. Zhang, "Global convergence of the nonmonotone MBFGS method for nonconvex unconstrained minimization," Journal of Computational and Applied Mathematics, vol. 223, no. 1, pp. 40-47, 2009.

[8] W. Zhou and X. Chen, "Global convergence of a new hybrid Gauss-Newton structured BFGS method for nonlinear least squares problems," SIAM Journal on Optimization, vol. 20, no. 5, pp. 2422-2441, 2010.

[9] D.-H. Li and M. Fukushima, "A modified BFGS method and its global convergence in nonconvex minimization," Journal of Computational and Applied Mathematics, vol. 129, no. 1-2, pp. 15-35, 2001.

[10] D.-H. Li and M. Fukushima, "On the global convergence of the BFGS method for nonconvex unconstrained optimization problems," SIAM Journal on Optimization, vol. 11, no. 4, pp. 1054-1064, 2001. 
[11] L. Liu, Z. Wei, and X. Wu, "The convergence of a new modified BFGS method without line searches for unconstrained optimization or complexity systems," Journal of Systems Science and Complexity, vol. 23, no. 4, pp. 861-872, 2010.

[12] Y. Xiao, Z. Wei, and Z. Wang, "A limited memory BFGS-type method for large-scale unconstrained optimization," Computers \& Mathematics with Applications, vol. 56, no. 4, pp. 1001-1009, 2008.

[13] C. Zhu, R. H. Byrd, P. Lu, and J. Nocedal, "Algorithm 778: 1-BFGS-B: fortran subroutines for large-scale bound-constrained optimization," ACM Transactions on Mathematical Software, vol. 23, no. 4, pp. 550-560, 1997.

[14] W. Zhou, "A modified BFGS type quasi-Newton method with line search for symmetric nonlinear equations problems," Journal of Computational and Applied Mathematics, vol. 367, Article ID 112454, 2020.

[15] L. Zhang and H. Tang, "A hybrid MBFGS and CBFGS method for nonconvex minimization with a global complexity bound," Pacific Journal of Optimization, vol. 14, no. 4, pp. 693-702, 2018.

[16] W. Zhou and L. Zhang, "A modified Broyden-like quasiNewton method for nonlinear equations," Journal of Computational and Applied Mathematics, vol. 372, Article ID 112744, 2020.

[17] M. J. D. Powell, "Some global convergence properties of a variable metric algorithm for minimization without exact line searches," SIAM-AMS Proceedings, vol. 9, pp. 53-72, 1976.

[18] R. H. Byrd, J. Nocedal, and Y.-X. Yuan, "Global convergence of a cass of quasi-Newton methods on convex problems," SIAM Journal on Numerical Analysis, vol. 24, no. 5, pp. 1171-1190, 1987.

[19] L. C. W. Dixon, "Variable metric algorithms: necessary and sufficient conditions for identical behavior of nonquadratic functions," Journal of Optimization Theory and Applications, vol. 10, no. 1, pp. 34-40, 1972.

[20] A. Griewank, "The global convergence of partitioned BFGS on problems with convex decompositions and Lipschitzian gradients," Mathematical Programming, vol. 50, no. 1-3, pp. 141-175, 1991.

[21] M. J. D. Powell, "On the convergence of the variable metric algorithm," IMA Journal of Applied Mathematics, vol. 7, no. 1, pp. 21-36, 1971.

[22] W. F. Mascarenhas, "The BFGS method with exact line searches fails for non-convex objective functions," Mathematical Programming, vol. 99, no. 1, pp. 49-61, 2004.

[23] Y.-H. Dai, "Convergence properties of the BFGS algorithm," SIAM Journal on Optimization, vol. 13, no. 3, pp. 693-701, 2006.

[24] G. Yuan and Z. Wei, "Convergence analysis of a modified BFGS method on convex minimizations," Computational Optimization and Applications, vol. 47, no. 2, pp. 237-255, 2010.

[25] J. Nocedal, "Theory of algorithms for unconstrained optimization," Acta Numerica, vol. 1, pp. 199-242, 1992.

[26] S. S. Oren and D. G. Luenberger, "Self-scaling variable metric (SSVM) algorithms, part I: criteria and sufficient conditions for scaling a class of algorithms," Management Science, vol. 20, no. 5, pp. 845-862, 1974.

[27] J. Nocedal and Y.-X. Yuan, "Analysis of self-scaling quasiNewton method," Mathematical Programming, vol. 61, no. 1-3, pp. 19-37, 1993.

[28] M. Al-Baali, "Analysis of a family of self-scaling quasi-Newton methods," Technical report, Department of Mathematics and
Computer Science, United Arab Emirates University, Al Ain, UAE, 1993.

[29] Y.-X. Yuan, "A modified BFGS algorithm for unconstrained optimization," IMA Journal of Numerical Analysis, vol. 11, no. 3, pp. 325-332, 1991.

[30] M. J. D. Powell, "How bad are the BFGS and DFP methods when the objective function is quadratic?" Mathematical Programming, vol. 34, no. 1, pp. 34-47, 1986.

[31] J. Barzilai and J. M. Borwein, "Two-point step size gradient methods," IMA Journal of Numerical Analysis, vol. 8, no. 1, pp. 141-148, 1988.

[32] W. Y. Cheng and D. H. Li, "Spectral scaling BFGS method," Journal of Optimization Theory and Applications, vol. 146, no. 2, pp. 305-319, 2010.

[33] N. Andrei, "An adaptive scaled BFGS method for unconstrained optimization," Numerical Algorithms, vol. 77, no. 2, pp. 413-432, 2017.

[34] N. Andrei, "A double parameter scaled BFGS method for unconstrained optimization," Journal of Computational and Applied Mathematics, vol. 332, pp. 26-44, 2018.

[35] Y.-H. Dai and C.-X. Kou, "A nonlinear conjugate gradient algorithm with an optimal property and an improved wolfe line search," SIAM Journal on Optimization, vol. 23, no. 1, pp. 296-320, 2013.

[36] Z. Dai, X. Dong, J. Kang, and L. Hong, "Forecasting stock market returns: new technical indicators and two-step economic constraint method," The North American Journal of Economics and Finance, vol. 53, Article ID 101216, 2020.

[37] Z. Dai and H. Zhu, "A modified Hestenes-Stiefel-type derivative-free method for large-scale nonlinear monotone equations," Mathematics, vol. 8, no. 2, p. 168, 2020.

[38] G. Yuan, X. Wang, and Z. Sheng, "Family weak conjugate gradient algorithms and their convergence analysis for nonconvex functions," Numerical Algorithms, vol. 84, no. 3, pp. 935-956, 2020.

[39] G. Yuan, J. Lu, and Z. Wang, “The PRP conjugate gradient algorithm with a modified WWP line search and its application in the image restoration problems," Applied Numerical Mathematics, vol. 152, pp. 1-11, 2020.

[40] G. Yuan, T. Li, and W. Hu, “A conjugate gradient algorithm for large-scale nonlinear equations and image restoration problems," Applied Numerical Mathematics, vol. 147, pp. 129-141, 2020.

[41] G. Yuan, Z. Wei, and Y. Yang, "The global convergence of the Polak-Ribière-Polyak conjugate gradient algorithm under inexact line search for nonconvex functions," Journal of Computational and Applied Mathematics, vol. 362, pp. 262275, 2019.

[42] L. Zhang, "A derivative-free conjugate residual method using secant condition for general large-scale nonlinear equations," Numerical Algorithms, vol. 83, no. 4, pp. 1277-1293, 2020.

[43] W. Zhou, "A short note on the global convergence of the unmodified PRP method," Optimization Letters, vol. 7, no. 6, pp. 1367-1372, 2013.

[44] W. Sun and Y. Yuan, Optimization Theory and Methods, Springer US, New York, NY, USA, 2006.

[45] R. H. Byrd, D. C. Liu, and J. Nocedal, "On the behavior of broyden's class of quasi-Newton methods," SIAM Journal on Optimization, vol. 2, no. 4, pp. 533-557, 1992.

[46] M. J. D. Powell, "Updating conjugate directions by the BFGS formula," Mathematical Programming, vol. 38, no. 1, pp. 29-46, 1987.

[47] I. Bongartz, A. R. Conn, N. Gould, and P. L. Toint, "CUTE: constrained and unconstrained testing environment," $A C M$ 
Transactions on Mathematical Software, vol. 21, no. 1, pp. 123-160, 1995.

[48] J. J. Moré, B. S. Garbow, and K. E. Hillstrom, "Testing unconstrained optimization software," ACM Transactions on Mathematical Software (TOMS), vol. 7, no. 1, pp. 17-41, 1981.

[49] Y. Yuan and W. Sun, Theory and Methods of Optimization, Science Press of China, Beijing, China, 1999.

[50] A. Ouyang, L.-B. Liu, Z. Sheng, and F. Wu, "A class of parameter estimation methods for nonlinear Muskingum model using hybrid invasive weed optimization algorithm," Mathematical Problems in Engineering, vol. 2015, Article ID 573894, 15 pages, 2015.

[51] A. Ouyang, Z. Tang, K. Li, A. Sallam, and E. Sha, "Estimating parameters of Muskingum model using an adaptive hybrid PSO algorithm," International Journal of Pattern Recognition and Artificial Intelligence, vol. 28, pp. 1-29, 2014.

[52] Z. W. Geem, "Parameter estimation for the nonlinear Muskingum model using the BFGS technique," Journal of Irrigation and Drainage Engineering, vol. 132, no. 5, pp. $474-478,2006$. 